correlacionada negativamente com as concentrações de MDA nos pacientes com dessas desordens. Os resultados deste estudo sugerem que uma formação aumentada de espécies reativas pode estar envolvida com a redução da atividade da BuChE nas desordens do metabolismo do propionato e que o tratamento com L-carnitina e dieta hipoprotéica é capaz de prevenir estas alterações. Apoio Financeiro: FIPE/HCPA, FAPERGS.

\title{
THE ASSOCIATION EFFECT OF INSULIN AND CLONAZEPAM ON OXIDATIVE STRESS IN LIVER OF EXPERIMENTAL ANIMAL MODEL OF DIABETES AND DEPRESSION
}

Carlos Alberto Yasin Wayhs, Caroline Tortato, Caroline Paula Mescka, Matheus Augusto de Bittencourt Pasquali, Carlos Eduardo Schnorr, Maurício Schuler Nin, Helena Maria Tannhauser Barros, Jose Claudio Fonseca Moreira, Carmen Regla Vargas

Introduction: Oxidative stress plays an important role in the development of diabetes complications, since hyperglycemia generates abnormally high levels of free radicals. Moreover, depression prevalence in diabetic patients is much higher than in the general population, and clonazepam is being used to treat this complication. It is known that there is oxidative stress in peripheral blood in experimental animal model of diabetes and depression, and acute treatment with insulin and clonazepam has a protective effect on oxidative stress in this model. Objective: Evaluate the effect of insulin plus clonazepam on oxidative stress parameters in liver of diabetic male rats induced with streptozotocin and submitted to forced swimming test (FST). Materials and Methods: Diabetes was induced by a single i.p. dose of Streptozotocin (STZ) $60 \mathrm{mg} / \mathrm{kg}$. Insulin (4 IU/ $\mathrm{kg}$ ) plus CNZ acute i.p. treatment $(0.25 \mathrm{mg} / \mathrm{kg})$ was administered 24,5 and 1 hour before the FST. The protein oxidative damage was evaluated by carbonyl formation. The antioxidant status was measured by the analyses of enzymatic activities of the superoxide dismutase (SOD) and catalase (CAT); The activity of the enzyme glyoxalase and glycemia levels also were determined. Results and Conclusions: There is protein oxidative damage in liver from diabetic rats submitted to FST, which was reverted by the treatment. The activities of the antioxidant enzymes were significantly decreased in liver from these animals, which was reverted by the treatment. So, insulin plus clonazepam treatment ameliorate the antioxidant status and protect against protein oxidative damage in liver of diabetic rats submitted to FST.

\section{A AÇÃo DO EXTRATO DE BOSWELLIA SERRATA EM MODELO EXPERIMENTAL DE COLITE}

Renata Minuzzo Hartmann, Francielli Licks, Elizângela Gonçalves Schemitt, Maria Isabel Morgan Martins, Josieli Raskopf Colares, Luise Meurer, Henrique Sarubbi Fillmann, Norma Anair Possa Marroni

A colite ulcerativa é classificada como uma doença inflamatória intestinal. O aumento de espécies reativas de oxigênio (ERO) e nitrogênio estão envolvidos na atividade da doença. O objetivo do estudo foi avaliar a pressão anal esfincteriana, os metabólitos do óxido nítrico (NO), a lipoperoxidação (LPO) e modificações histológicas e imunohistoquímicas. Foram utilizados 22 ratos machos Wistar $( \pm 350 \mathrm{~g})$ divididos em: 1: Controle (CO), 2: Controle+B. serrata $(C O+B)$; 3: Colite $(C L)$, 4: Colite+B. serrata $(C L+B)$. O extrato da planta Boswellia serrata (B. serrata) $(34,2 \mathrm{mg} / \mathrm{Kg})$ foi administrado uma vez ao dia durante 48 horas após a indução da colite por ácido acético (4\% via enema). Foi aferida a pressão anal esfincteriana, avaliação da LPO por TBARS, dos metabólitos do NO por nitritos e nitratos e análise histológica por hemotoxilina e eosina (HE) e imunohistoquímica da enzima óxido nítrico sintase induzível (iNOS). A análise estatística foi através de ANOVA seguido do teste StudentNewman-Keuls (mÈdia $\pm E P$ ) com significância quando $\mathrm{p}<0,05$. A pressão anal esfincteriana $(\mathrm{cm} / \mathrm{H} 2 \mathrm{O})$ do grupo

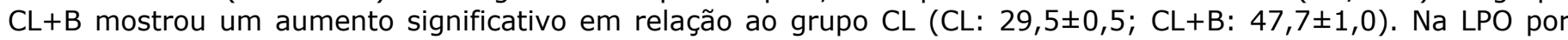
TBARS (nmoles/mgprot) ocorreu uma diminuição significativa no grupo $\mathrm{CL}+\mathrm{B}$ em relação ao grupo $\mathrm{CL}$ (CL:

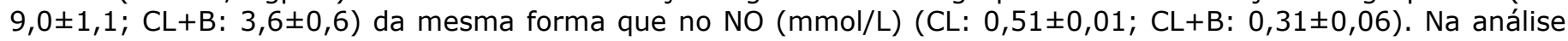
histológica e de imunohistoquímica o grupo $C L+B$ apresentou uma diminuição de edema, da inflamação e da presença da iNOS. Os dados sugerem que o extrato de B. serrata tem um efeito protetor contra as ERO diminuindo a LPO e ação anti-inflamatória, a julgar pelo aumento da pressão anal esfincteriana e pela redução do NO, edema e inflamação. Apoio: FIPE (11-0215) / CAPES / ULBRA.

\section{LESÃo PULMONAR AgUdA EXPERIMENTAL PROMOVE MUdANÇAS NEUROQUímicas em CóRteX CEREBRAL DE RATOS}

Eduardo Peil Marques, Maira Jaqueline da Cunha, Aline Andrea da Cunha, Emilene Barros da Silva Scherer, Samanta Oliveira Loureiro, Fernanda Rossatto Machado, Felipe Schmitz, Angela Terezinha de Souza Wyse

A lesão pulmonar aguda (LPA) e a síndrome da angustia respiratória (SARA) são caracterizadas por inflamação pulmonar aguda e persistente. Estudos demonstram que pacientes com SARA podem apresentar disfunções neurológicas e cognitivas após a alta hospitalar. Entretanto os mecanismos envolvidos em tais alterações são pouco compreendidos. O objetivo do presente estudo foi investigar as possíveis alterações neuroquímicas e inflamatórias em córtex cerebral de ratos submetidos à LPA. Ratos Wistar de 90 dias foram divididos em 2 grupos: controle (salina) e LPA (injeção intratraqueal de LPS na dose de $100 \mathrm{~g} / 100 \mathrm{~g}$ de peso corporal). Doze horas após a lesão os animais foram mortos e o córtex cerebral foi dissecado para avaliação dos parâmetros de estresse oxidativo (níveis de espécies reativas, dano a lipídeos e proteínas, atividade das enzimas antioxidantes, capacidade antioxidante total, níveis de glutationa e atividade da glicose-6-fosfato desidrogenase), níveis de 